\begin{tabular}{|l|l|l|}
\hline R.D. & Raj D. & Sheth $^{\mathrm{a}, \mathrm{b}}$ \\
\hline J.E. & Jack E. & Riggs $^{\mathrm{a}, \mathrm{b}, \mathrm{c}}$ \\
\hline O.A. & Orlando A. & Ortiz $^{\mathrm{a}, \mathrm{e}}$ \\
\hline
\end{tabular}

Departments of ${ }^{a}$ Neurology, ${ }^{\mathrm{b}}$ Pediatrics, ${ }^{\mathrm{C}}$ Community Medicine, ${ }^{\mathrm{d}}$ Medicine, and ${ }^{\mathrm{e}}$ Radiology West Virginia University Health Sciences Center, Morgantown, W. Va., USA,

\title{
Raymond Syndrome: A Validation
}

\section{Raj D. Sheth, MD, Assistant Professor of Pediatrics and Neurology, Box 9180, West Virginia University Health Sciences Center, Morgantown, WV $26506-9180$ (USA)}

Fulgence Raymond, Charcot's successor at the Salpetriere, is most remembered for his clinico-pathologic description of the Ray-mond-Cestan syndrome

(chorea, hemianesthesia and tremor) [1]. In a lecture on January 11, 1895, Raymond [2] described a 39-year-old syphilitic woman with an abducens palsy and contralateral central facial paresis and hemiparesis. Raymond suggested that a circumscribed lesion at the facial decussation in the basis of the medial caudal pons could produce this clinical syndrome. After reviewing the original description, Wolfe stated that 'Raymond's patient clearly did not have the syndrome, since she developed a cerebral right hemiplegia, with aphasia and difficulty recognizing her husband's face. This was followed three months later by a left abducens palsy' [3]. There are few, if any, examples of true Raymond syndrome in the literature. For example, a recently reported patient with Raymond syndrome did not have facial weakness [4].

A 55-year-old black woman, with a 10-year history of hypertension, experienced the sudden onset of right-sided weakness and diplopia. On neurological examination, she was alert, oriented and
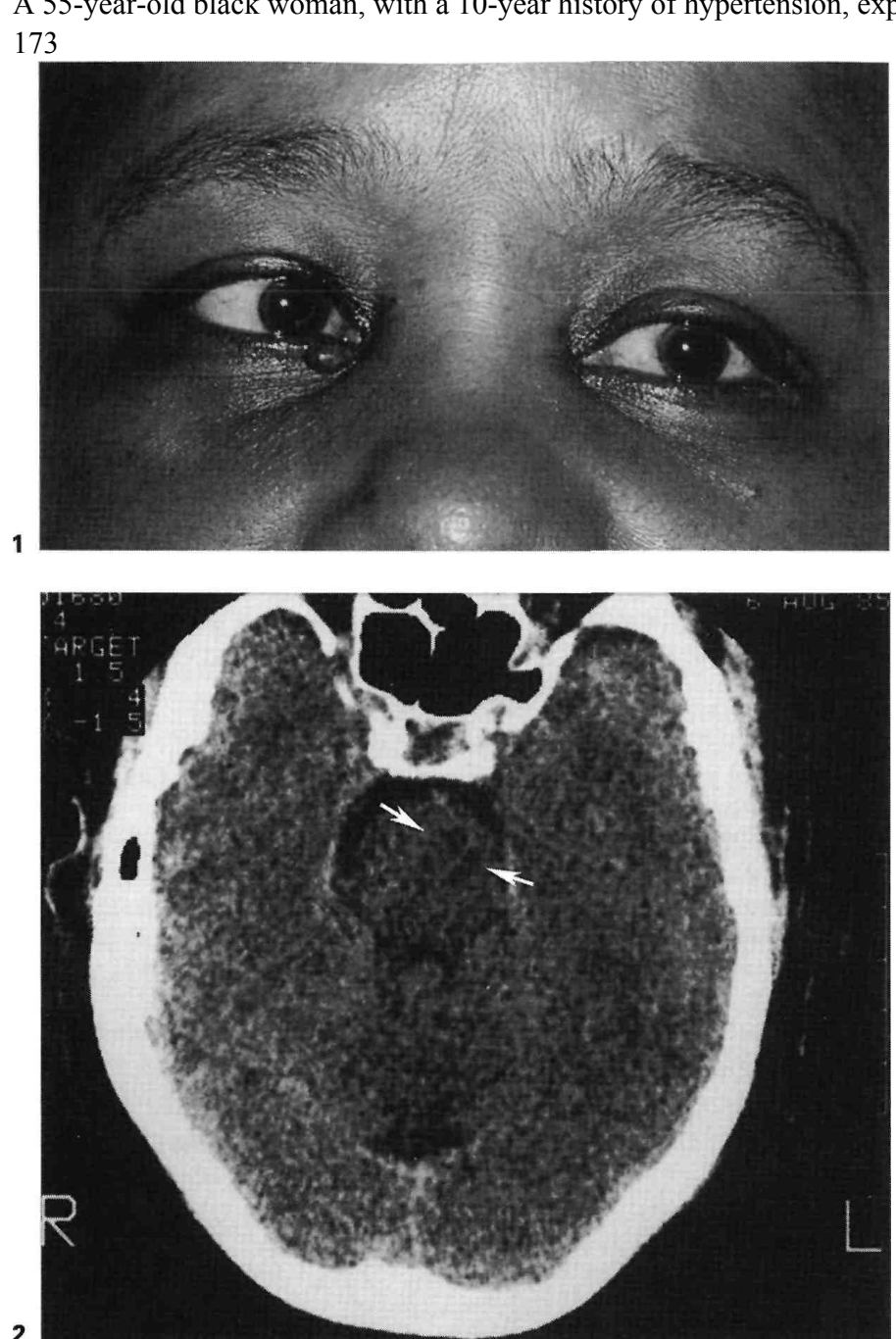

Fig. 1. When asked to look to the left, the patient could not move the left eye past midline in abduction.

Fig. 2. Cerebral computed tomography with 1.5 -mm slices through the brain stem demonstrated a circumscribed area of low attenuation in the basis of the left medial caudal pons (arrows)

had normal language function. She was unable to move the left eye laterally past the midline (fig. 1) and had a right central facial paresis. The remainder of her cranial nerve examination was normal. She also had minimal movement of her right upper and lower extremity. Sensation was normal.

Cranial computed tomography on admission was normal. Repeat scan, 8 days later, showed a small focal nonenhancing low-density lesion in the basis of the medial caudal pons on the left (fig. 2), consistent with a lacunar infarction. A magnetic resonance imaging (MRI) study obtained 10 years after this stroke showed resolution of the lacunar infarct. No other lesion was identified on the MRI that would account for her a lacunar infarction. A magnetic resonance imaging (MRI) study obtained 10 years after this stroke showed resolution of the lacunar infarct. No other lesion was identified on the MRI that would accoin
initial clinical syndrome or her persistent right hemi-paresis. During the 10 -year follow-up period, her right hemiparesis persisted, and she had no clinical episodes suggestive of a subsequent stroke.

The computed tomographic location of the small infarction in the basis of the medial caudal pons coincides with Wolfe's illustration of the lesion that could produce Raymond syndrome [4]. The unusual combination of abducens palsy and contralateral central facial paresis in Raymond syndrome implies that the facial corticobulbar decussation must occur very close to the level of the sixth nerve in the pons. In accordance with this clinical neuroanatomical deduction, Kupers [5] has reported pathological evidence that the facial decussation is located in the pons at the level of the facial nuclei. 


\section{References}

McHenry LC: Garrison's History of Neurology. Springfield, Thomas, 1969, pp 291-293.

Raymond F: Concerning a special type of alternating hemiplegia; in Lecons sur les maladies nerveuses 1894-1895, Ire ser. Paris, Ricklin \& Soques, 1896, pp 365-383.

Wolfe JK: Raymond's syndrome. The Classic Brain Stem Syndromes (transl of the original papers with notes on the evolution of clinical neuroanatomy). Springfield, Thomas, 1936 , pp 139-141.

Satake M, Kira J, Yamada T, Kobayashi T: Raymond syndrome (alternating abducent hemiplegia) caused by a small hematoma at the medial pon-tomedullary junction. J Neurol Neurosurg Psychiatry

1995;58:261.

Kupers HGJM: Corticobulbar connections to the pons and the lower brainstem in man: An anatomical study. Brain 1958;81:364-388. 